\title{
Incidental discovery of a rectal polypoid lesion
}

\author{
Hyun Seok Lee
}

Department of Internal Medicine, Kyungpook National University Hospital, School of Medicine, Kyungpook National University, Daegu, Korea

Question: A 40-year-old woman underwent screening colonoscopy at another hospital. She had no abdominal symptoms. Colonoscopy showed multiple polypoid lesions at the proximal rectum. Pathologic results of forceps biopsy showed a hyperplastic polyp with surface ulceration. She was transferred to the outpatient clinic at Kyungpook National University Hospital for further evaluation and management. Because malignancy could not be ruled out, it was decided that she undergo polypectomy on the polypoid lesion (Fig. A) for pathologic diagnosis. During colonoscopy, submucosal saline was injected before snaring, and the lesion showed a positive nonlifting sign. However, endoscopic mucosal resection (EMR) was performed (Fig. B) to identify pathologic features despite incomplete resection (Fig. C). What is the most likely diagnosis?

\section{Answer: Colorectal Endometriosis}

The pathologic diagnosis of the EMR specimen was colorectal endometriosis (Fig. D, H\&E, $\times 40$ ). Endometriosis is defined as the presence of endometrial glands and stroma at extrauterine sites including the pelvis. The preoperative diagnosis can oc- casionally be challenging due to the various clinical manifestations. Endometriosis involves the colon and rectum in about $5 \%$ of cases. Colorectal endometriosis lesions can be deeply infiltrating. Most colorectal lesions do not infiltrate the full thickness of the colorectal wall. In a systematic review of patients who underwent large bowel resection for colorectal endometriosis, $38 \%$ of lesions penetrated to the submucosa and only $6 \%$ invaded the mucosa. ${ }^{1}$

Sigmoidoscopy or colonoscopy is rarely useful to diagnose bowel endometriosis as lesions that penetrate the mucosa are unusual. It is, however, important to perform colonoscopy prior to surgery in order to exclude a malignancy if there are symptoms suggestive of a bowel neoplasm and to assess possible colorectal stricture in women with symptoms suggestive of partial bowel obstruction., ${ }^{2,3}$ In a study of 83 women undergoing surgical resection for bowel endometriosis, 8\% (7/83) had a concurrent malignancy. ${ }^{3}$

It can sometimes be difficult to differentiate between colorectal endometriosis and colon cancer or other conditions. Polypoid colorectal endometriosis is particularly diffi-
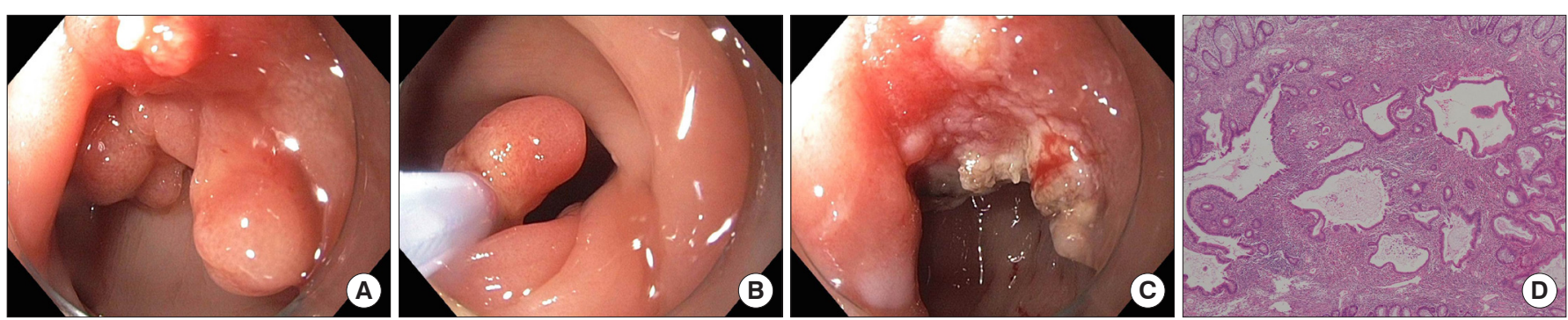

Received September 17, 2018. Revised September 21, 2018. Accepted September 22, 2018.

Correspondence to Hyun Seok Lee, Department of Internal Medicine, Kyungpook National University Hospital, School of Medicine, Kyungpook National University, 807 Hoguk-ro, Buk-gu, Daegu 41404, Korea. Tel: +82-53-200-2603, Fax: +82-53-200-2027, E-mail: Ihsworld@nate.com 
cult to distinguish from a colorectal tumor. Endometriosis is definitively diagnosed with histologic evaluation of a biopsied lesion. ${ }^{4}$ If mucosal abnormalities are identified during colonoscopy, targeted biopsies should be obtained. However, if the colorectal mucosa is not involved, an accurate diagnosis cannot be made with a colonoscopic biopsy. Therefore, normal biopsy results may not rule out colorectal endometriosis and further evaluation may be needed.

In this case, pelvic MRI did not provide additional information on the patient's colorectal endometriosis. Although abdominal CT or MRI is an important tool in preoperative evaluation of colorectal endometriosis, a surgical procedure can help make a tissue-based diagnosis if a colonoscopy fails to offer a pathologic diagnosis. Diagnostic laparoscopy or laparotomy can help to diagnose and confirm endometriosis pathologically. $^{3}$

\section{FINANCIAL SUPPORT}

The author received no financial support for the research, authorship, and/or publication of this article.

\section{CONFLICT OF INTEREST}

No potential conflict of interest relevant to this article was reported.

\section{AUTHOR CONTRIBUTION}

Hyun Seok Lee selected the case, wrote the manuscript, processed the images and pathologic findings, and reviewed the final version of the manuscript.

\section{REFERENCES}

1. Meuleman C, Tomassetti C, D'Hoore A, et al. Surgical treatment of deeply infiltrating endometriosis with colorectal involvement. Hum Reprod Update 2011;17:311-326.

2. Remorgida V, Ferrero S, Fulcheri E, Ragni N, Martin DC. Bowel endometriosis: presentation, diagnosis, and treatment. Obstet Gynecol Surv 2007;62:461-470.

3. Kaufman LC, Smyrk TC, Levy MJ, Enders FT, Oxentenko AS. Symptomatic intestinal endometriosis requiring surgical resection: clinical presentation and preoperative diagnosis. Am J Gastroenterol 2011;106:1325-1332.

4. Kanthimathinathan V, Elakkary E, Bleibel W, Kuwajerwala N, Conjeevaram S, Tootla F. Endometrioma of the large bowel. Dig Dis Sci 2007;52:767-769. 